OPEN ACCESS

Edited by:

Kay Tetzlaff,

University Hospital of Tübingen,

Germany

Reviewed by:

Andreas Koch,

Christian-Albrechts-University,

Germany

Jacek Kot

Medical University of Gdańsk, Poland

*Correspondence:

Costantino Balestra

costantinobalestra@gmail.com

Specialty section:

This article was submitted to Environmental, Aviation and Space

Physiology,

a section of the journal

Frontiers in Physiology

Received: 23 August 2021 Accepted: 07 October 2021

Published: 29 October 2021

Citation:

Germonpré $P$, Lafère $P$, Portier W,

Germonpré F-L, Marroni A and

Balestra C (2021) Increased Risk of Decompression Sickness When

Diving With a Right-to-Left Shunt:

Results of a Prospective

Single-Blinded Observational Study

(The "Carotid Doppler" Study).

Front. Physiol. 12:763408.

doi: 10.3389/fphys.2021.763408

\section{Increased Risk of Decompression Sickness When Diving With a Right-to-Left Shunt: Results of a Prospective Single-Blinded Observational Study (The "Carotid Doppler" Study)}

\author{
Peter Germonpré ${ }^{1,2}$, Pierre Lafère ${ }^{1,2,3}$, William Portier ${ }^{1}$, Faye-Lisa Germonpré1, \\ Alessandro Marroni ${ }^{3}$ and Costantino Balestra ${ }^{2,3,4 *}$
}

${ }^{1}$ Centre for Hyperbaric Oxygen Therapy, Military Hospital, Brussels, Belgium, ${ }^{2}$ Divers Alert Network (DAN) Europe Research Division, Roseto, Italy, ${ }^{3}$ Environmental \& Occupational, Ageing (Integrative) Physiology Lab, Haute Ecole Bruxelles-Brabant (HE2B), Auderghem, Belgium, ${ }^{4}$ Physical Activity Teaching Unit, Motor Sciences Department, Université Libre de Bruxelles (ULB), Brussels, Belgium

Introduction: Divers with a patent Foramen Ovale (PFO) have an increased risk for decompression sickness (DCS) when diving with compressed breathing gas. The relative risk increase, however, is difficult to establish as the PFO status of divers is usually only determined after a DCS occurrence.

Methods: This prospective, single-blinded, observational study was designed to collect DCS data from volunteer divers after screening for right-to-left shunt (RLS) using a Carotid Doppler test. Divers were blinded to the result of the test, but all received a standardized briefing on current scientific knowledge of diving physiology and "lowbubble" diving techniques; they were then allowed to dive without restrictions. After a mean interval of 8 years, a questionnaire was sent collecting data on their dives and cases of DCS (if any occurred).

Results: Data was collected on 148 divers totaling 66,859 dives. There was no significant difference in diving data between divers with or without RLS. Divers with RLS had a 3.02 times higher incidence of (confirmed) DCS than divers without RLS $(p=0.04)$. When all cases of (confirmed or possible DCS) were considered, the Relative Risk was $1.42(p=0.46)$. DCS occurred mainly in divers who did not dive according to "low-bubble" diving techniques, in both groups.

Conclusion: This prospective study confirms that DCS is more frequent in divers with RLS (such as a PFO), with a Relative Risk of 1.42 (all DCS) to 3.02 (confirmed DCS). It appears this risk is linked to diving behavior, more specifically diving to the limits of the adopted decompression procedures.

Keywords: decompression sickness, prospective study, relative risk, right-to-left shunt (RLS), patent foramen ovale (PFO), adverse effects, SCUBA dive, diving 


\section{INTRODUCTION}

Since the early 1990's, patency of the Foramen Ovale of the heart (Patent Foramen Ovale, PFO) has been identified as a risk factor for decompression sickness (DCS) in selfcontained underwater (SCUBA) diving (Moon et al., 1989; Wilmshurst et al., 1989). Numerous case reports have illustrated so-called "undeserved DCS," DCS occurring after dives within the accepted decompression limits and without violation of accepted decompression procedures, to be associated with PFO (Germonpre et al., 1998; Sykes and Clark, 2013). Several attempts have been made to quantify the increased risk of diving for divers with a PFO based on retrospective diving accident data (Bove, 1998; Torti et al., 2004). In these "risk-comparison" papers, a 2.9 (Bove, 1998) to 5.7 times higher risk (Torti et al., 2004) for DCS has been reported, although the latter figure has been criticized based on the subjective definition criteria used for DCS (Germonpre and Balestra, 2004).

This retrospective approach is only a rough approximation of the actual increase of the risk, for several reasons. First, because the total number of dives performed is not known, the denominator of the risk equation is missing. Secondly, the type of diving influences the risk for DCS, with certain diving behavior such as deep decompression diving, technical diving - yielding a significantly higher risk than no-decompression recreational diving (Germonpre, 2006). Third, DCS is characterized by a highly variable spectrum of symptoms, making the diagnosis often difficult; both over-reporting and underreporting are frequently observed (Steffensmeier et al., 2017; Hubbard et al., 2018). Lastly, the diagnostic accuracy of PFO detection is highly dependent on the technology and technique used for PFO detection (Gin et al., 1993; Wilmshurst et al., 2003; Attaran et al., 2006; Johansson et al., 2010). Unless all these factors can be accounted for, estimates of increased DCS risk remain highly speculative (Vann et al., 2008; Wilmshurst, 2019).

With the increased availability and documented safety of percutaneous closure devices for PFO, interventional therapy appeals to more and more divers as a simple and safe cure for "the PFO problem." Indeed, studies have shown that closing the PFO reduces the risk of paradoxical embolization of decompression bubbles (Honěk et al., 2014a) and also of decompression sickness (Billinger et al., 2011; Honěk et al., 2020). On the other hand, adhering to more "conservative" diving profiles has also been shown to reduce the risk, in a similar degree (Klingmann et al., 2012; Honěk et al., 2014b). Percutaneous PFO closure carries a small but non-negligible risk of procedural complications, as well as significant costs to be carried by the patient or by public money (Social Security); adopting safer diving profiles mainly restricts the recreational diver psychologically (the feeling of "being limited"). Generally, PFO closure in the context of diving is not regarded as a medical necessity, except perhaps for professional and military divers (Pristipino et al., 2021).

In order to help divers and diving medicine physicians decide which approach is the best in a particular case, determining the relative risk in a more precise way, is important. We aimed to perform a prospective, single-blinded study to determine the relative risk (RR) of diving with a right-to-left shunt (RLS).

\section{MATERIALS AND METHODS}

\section{General Methodology}

The feasibility of such a study needed to be ascertained first. A power analysis was made based on available retrospective data. We had to develop a screening test that we could use on a large number of divers without any problems. We had to address the fact that if we test divers and we tell them whether they have a right-to-left shunt or not, they might change their diving behavior and so "falsify" the study by diving much safer then they normally would. Inversely, divers who were told they do not have a shunt might feel less "vulnerable" and less restricted to perform more "risky" dives (in an extreme scenario, we could end up seeing divers without RLS having more DCS than divers with RLS). Ethical committee approval was needed, not only for the screening test but also for the fact that divers would be blinded to the result of the test. We had to take into account the probability of a large number of drop-outs ("lost to follow-up") as the study would take years to complete. Finally, we would have to make sure that data were collected with a maximum of accuracy, both regarding exposure (number and types of dives performed) and outcome (absence or presence of DCS).

\section{Sample Size Calculations and Statistics}

We assumed the Odds Ratio for DCS when having RLS to be 4, which is higher than reported by Bove (1998) but lower than was later reported in the Swiss study (Torti et al., 2004). We assumed the prevalence of PFO in the general divers' population to be 25 percent, as reported in the seminal Mayo Clinic autopsy study (Hagen et al., 1984). Even though a PFO seems to be more prevalent in the young and less prevalent in older people, having a single rounded number is convenient for these sample size calculations.

We assumed a general risk of DCS for divers without a PFO to be 1 in 10,000 dives. For our "best case scenario," with a 95\% power of the study and no dropouts at the end of the study, we needed a total of 200,792 dives or 803 divers, each performing an average of 50 dives/year over 5 years. In a "worst case scenario" with a 50 percent dropout and a power of only $80 \%$, we would need 235,712 dives (or about 940 divers for a period of five years).

Results are analyzed with descriptive statistics, Student $t$-test after checking for normality, or proportion analysis where appropriate.

\section{Development of the Screening Test}

In order to be an acceptable screening test for RLS, it needs to be minimally invasive, low tech, low cost, and have a good sensitivity/specificity ratio. In 1999, we developed and described the Carotid Artery Doppler test (Germonpré et al., 1999; Wendling et al., 2001). In short, a large antecubital intravenous catheter was placed in the right elbow fold, and connected to a short tubing with two three-way valves attached. An intravenous injection of $9.5 \mathrm{~mL}$ of normal saline solution with $0.5 \mathrm{~mL}$ of air, agitated by pushing to and fro between two $10 \mathrm{~mL}$ syringes, was performed at the end of a respiratory "straining" maneuver. This is similar to the procedure used 
during a classic contrast echocardiography for detection of PFO. The "straining" maneuver (often erroneously called a Valsalva maneuver) consisted of a voluntary intrathoracic pressure increase (blocking the respiration and "bearing down") for ten seconds, followed by an abrupt release of pressure by exhaling. The agitated saline solution was rapidly injected just before release of the straining. Using an $8 \mathrm{MHz}$ vascular Doppler device placed over the left carotid artery, a "gurgling" bubble sound could be heard over the regular arterial Doppler sounds, in case of a RLS. The test subject was blinded to those sounds using headphones with loud music.

This test was validated on a group of 33 patients, in a single blinded comparison with contrast transesophageal echocardiography, and yielded a very good sensitivity (100\%) and specificity (88\%) with only a few "false positives" (as doubtful signals were to be classified positive) Wendling et al., 2001). A French group (Blatteau, 1999) replicated our validation test on 200 patients with transesophageal contrast echo, and found similar good figures for sensitivity and specificity (sensitivity $89 \%$, specificity 97\%).

During the development of the test, we verified that the sounds that are heard are actual bubbles passing into the carotid artery. Using 2-D duplex scanning, bubbles could be seen passing the beam of the Doppler probe and producing the distinctive sound (Figure 1).

\section{Recruitment of Investigators and Subjects}

A dedicated page on the DAN Europe website was set up to recruit investigators, who were all diving medicine physicians. We developed a study package consisting of a PowerPoint presentation, printed forms, report files, and injection materials, if needed. We organized "Carotid Artery Doppler" training workshops, because, even if the test was relatively easy to perform, training was needed to make sure there were no "false negatives." All the materials were developed in Dutch, French, English, German, and Italian, those being the primary target areas in Europe (in Europe, over 25 different languages are spoken, so we would only develop specific language materials if a sufficiently high yield was expected). During the workshop, every investigator received a hands-on training on about 10 divers to ascertain they could reliably report the results of the test. Furthermore, round the clock telephone and e-mail support was provided.

We used the DAN Europe website and personal contacts to recruit divers and organized "research sessions" for about 10 to 12 divers at a time. First, these divers were given a $1.5 \mathrm{~h}$ long lecture, illustrated by the Powerpoint presentation, on diving decompression risks. During this lecture, information was given on how the risk for DCS is dependent, not only on gas load but also on many other, often unknown factors; about PFO and how it would increase the risk for DCS; on the importance of conservative, "low-bubble" diving to reduce the risk of DCS. Then they were informed again that, while they would be tested for RLS, they would not be told the result of the test; and we would simply encourage them to "dive safely" (Wendling et al., 2001).

\section{Testing}

Ethical Committee approval was obtained (Bioethics Committee of the Belgian Defense Force Medical Staff, 2003), and divers signed an informed consent form prior to the testing. Each testing was performed individually, subjects going through the informed consent process one at a time. During the test, subjects wore headphones with loud music so they couldn't hear the Carotid Doppler signals. First, we did a few "simulated injections," to practice the straining maneuver. Then up to three saline injections with a properly performed straining maneuver were performed (Figure 2).

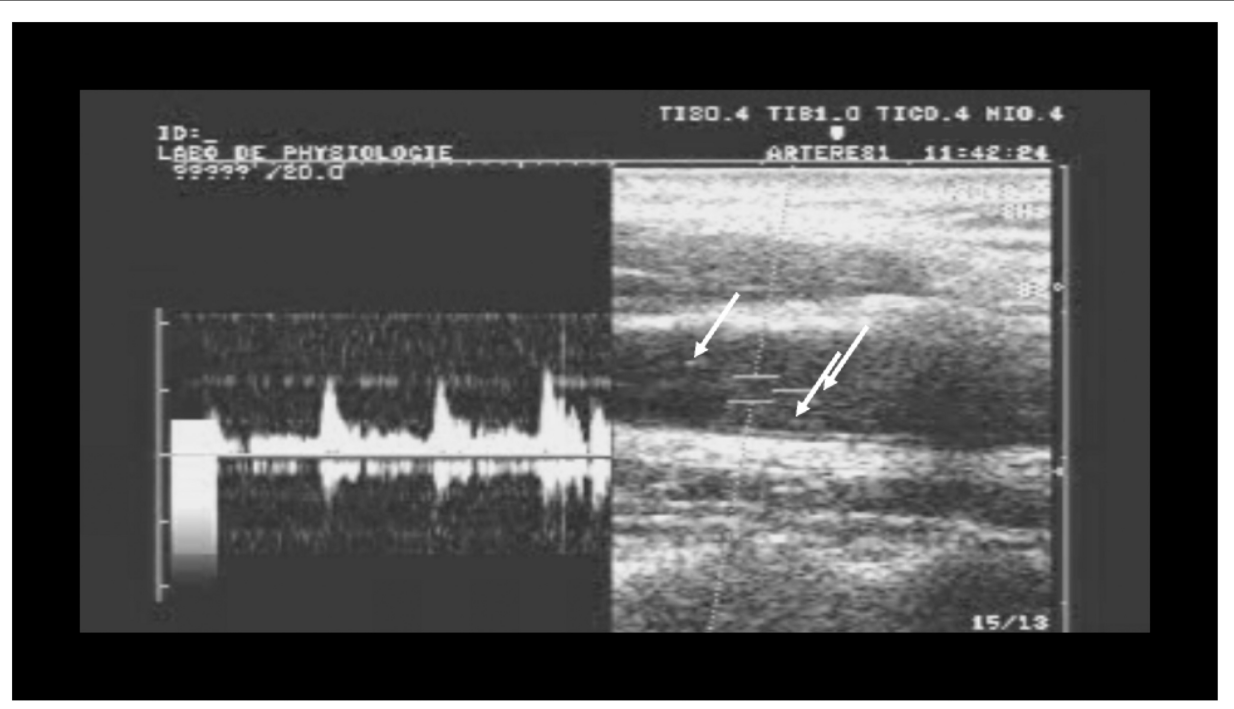

FIGURE 1 | Simultaneous Pulsed Wave Doppler-mode and B-mode ultrasound over the carotid artery, showing bubbles (arrows) passing through the imaging field producing the "gurgling" sound heard over the carotid artery with Doppler. 


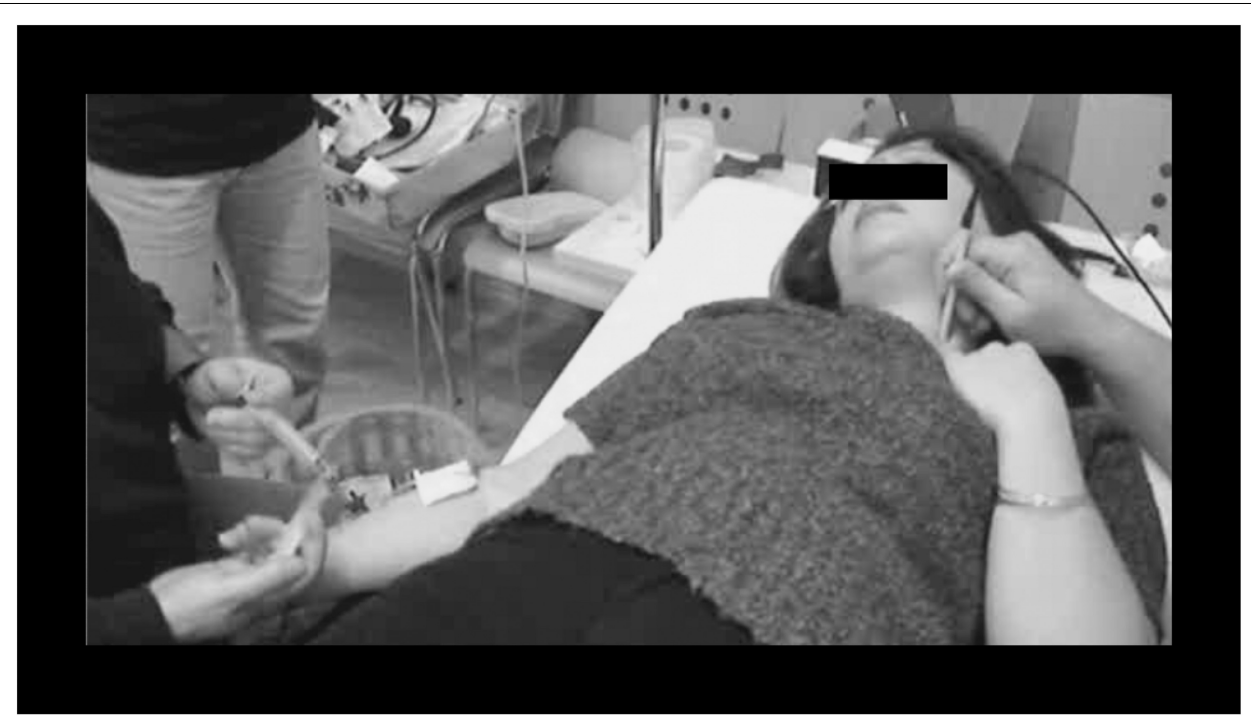

FIGURE 2 | Carotid Doppler test. Right arm: injection of contrast medium (10 mL of agitated saline solution); Left carotid artery: 8 MHz Doppler probe.

After the test, divers were again reminded of the importance of "safe diving" and were provided with a "Carotid Doppler Study Participant" card, with a telephone number and an e-mail address. They were asked to inform the investigators in case of a diving accident (but not as an emergency phone number) and also if they moved or changed telephone or e-mail address. They were also informed that approximately 5-6 years after the test, they would be contacted again to provide information on their dives and diving incidents or accidents.

\section{RESULTS}

The study had a recruitment period of about eight years (20012009). During that period, 11 investigators were recruited and trained, but only 6 have provided final data. Four provided data on less than 10 divers after their initial training. Three of the investigators had decided not to blind the divers to the result, thus deviating from the study protocol. These "non-blinded" data were analyzed separately.

Four hundred and forty-five divers were recruited, of which $55 \%$ were effectively blinded to the result. The mean age of the test subjects was 38.3 years, and $32 \%$ were female. In $18.9 \%$ of subjects, the Carotid Doppler test was positive, indicating a RLS. This was the initial data collection.

Each participating diver was contacted again after a period of between 7 and 10 years, and was sent a "final questionnaire" enquiring on their diving experience since the test (number and types of dives), the DCS or diving incidents they might have had, and a number of other questions to make the questionnaires both detailed but not too cumbersome to fill in. The questionnaire can be found in the Supplementary Materials.

As expected, a large number of divers could not be located anymore, even though telephone numbers, postal address, e-mail and other data had been recorded. Only 33.3 percent of participants returned the questionnaire (148 divers). Efforts to retrieve more questionnaires were continued through the end of 2019.

The majority of the return data were received from "blinded" divers. Of the "non-blinded" divers only $14.5 \%$ responded, as opposed to $46.12 \%$ of the "blinded." There were some divers who stopped diving after the test, and that may have been or may not have been because the test was positive. Two divers had their PFO closed after detecting it with the Carotid Doppler test.

The total number of dives collected was 66,859. Demographics (see Table 1) showed no differences between RLS-negative and RLS-positive divers. There were no significant differences in sex distribution, age, height and weight, smoking habits, or study duration (7.94 vs. 7.96 years). The diving experience before and the number of dives after the tests was not significantly different, although RLS positive divers tended to have slightly fewer dives before the test (441 vs. 524 dives, NS).

Most divers used dive computers for decompression management, most of them used the popular dive computer brands (Uwatec, Suunto). Some divers still used tables, some divers used "technical" dive computers, but the majority could be considered recreational divers (Table 2). This reflects the recreational diving population characteristics in the first decade of this century. In the final questionnaire we also inquired what kind of dives they had done during the study period. We arbitrarily divided the dives into "decompression air dives", "no-decompression air dives," "decompression nitrox dives," "no-decompression nitrox dives," "technical dives" and "nitrox on air tables/computer" dives, providing a detailed description of each type of diving activity. We also tried to make a second categorization of the dives, between "recreational dives," "sports dives" (deep square dives, such as on wrecks or deep reefs), "deep dives" (over 40 meters), and "low risk dives." Again, there is no significant difference between the two groups in this respect. 
TABLE 1 | Biometry and dive experience.

\begin{tabular}{|c|c|c|c|}
\hline $\mathbf{n}$ & $\frac{\text { RLS Positive }}{28}$ & $\frac{\text { RLS Negative }}{120}$ & p-value \\
\hline Males (\%) & 71.4 & 80.0 & ns \\
\hline Age (mean $\pm \mathrm{SD}$ ) & $35.0 \pm 8.7$ & $39.1 \pm 11.3$ & ns \\
\hline Smokers (\%) & 10.7 & 14.2 & ns \\
\hline Duration of study (years) & 8.0 & 7.9 & ns \\
\hline Height (cm, mean \pm SD) & $171.6 \pm 22.0$ & $178.5 \pm 12.5$ & ns \\
\hline Weight $(\mathrm{kg}$, mean $\pm \mathrm{SD})$ & $76.7 \pm 14.0$ & $82.7 \pm 24.7$ & ns \\
\hline $\begin{array}{l}\text { Dive experience before test } \\
\text { (number of dives) (mean } \pm \mathrm{SD} \text { ) }\end{array}$ & $441.0 \pm 751.2$ & $524.7 \pm 843.56$ & ns \\
\hline $\begin{array}{l}\text { Average number of Dives/year } \\
\text { before test (mean } \pm \mathrm{SD} \text { ) }\end{array}$ & $59.1 \pm 82.0$ & $55.4 \pm 36.1$ & ns \\
\hline Total number of dives since test & 15.494 & 51.365 & ns \\
\hline Total hours diving since test & 11.827 & 41.043 & ns \\
\hline $\begin{array}{l}\text { Dives/subject since test } \\
\text { (mean } \pm \mathrm{SD} \text { ) }\end{array}$ & $553,4 \pm 712.2$ & $428.0 \pm 467.6$ & ns \\
\hline
\end{tabular}

TABLE 2 | Type of diving performed during study period (for description of types of dives, see article text).

\begin{tabular}{lccc}
\hline & $\begin{array}{c}\text { RLS } \\
\text { Positive }\end{array}$ & $\begin{array}{c}\text { RLS } \\
\text { Negative }\end{array}$ & p-value \\
\hline Main dive computer used: UWATEC (\%) & 53.6 & 53.3 & $\mathrm{~ns}$ \\
Main dive computer used: SUUNTO (\%) & 39.3 & 28.3 & $\mathrm{~ns}$ \\
Main dive computer used: Other (\%) & 0.0 & 10.0 & $\mathrm{~ns}$ \\
Dive table use (\%) & 3.6 & 13.3 & $\mathrm{~ns}$ \\
Total number of dives since test & 15.494 & 51.365 & $\mathrm{~ns}$ \\
Decompression Air Dives (\%) & 20.10 & 23.67 & $\mathrm{~ns}$ \\
No Decomp Air Dives (\%) & 66.05 & 60.02 & $\mathrm{~ns}$ \\
Decompression Nitrox Dives (\%) & 3.02 & 3.47 & $\mathrm{~ns}$ \\
No Decomp Nitrox Dives (\%) & 5.97 & 7.62 & $\mathrm{~ns}$ \\
Nitrox Dives Using Air Tables (\%) & 1.56 & 2.95 & $\mathrm{~ns}$ \\
Other Breathing Gas/Technical diving (\%) & 5.12 & 5.75 & $\mathrm{~ns}$ \\
"Recreational” Dives & 27.53 & 30.98 & $\mathrm{~ns}$ \\
"Sports" Dives & 40.21 & 42.70 & $\mathrm{~ns}$ \\
"Deep" Dives & 22.50 & 19.68 & $\mathrm{~ns}$ \\
"Low Risk" Dives & 12.54 & 14.46 & $\mathrm{~ns}$ \\
\hline
\end{tabular}

A total of $8.3 \%$ of RLS negative divers and $28.6 \%$ of RLS positive divers had experienced a confirmed episode of DCS during the study period. The incidence of DCS per 10,000 dives was 1.95 and 5.16, respectively, yielding a Relative Risk of 2.65 (CI 1.05 to $6.72, p=0.039$ ). Some divers reported symptoms, not having been treated as DCS and a detailed description of these symptoms failed to positively identify those as DCS. These were classified as "possible DCS." If also those cases of "possible DCS" are taken into account, the incidence in RLS negative divers is $15.8 \%$ and in RLS positive divers $32.1 \%$. The incidence per 10,000 dives is a little more than 1.5 times higher for RLS positive divers (5.81/10,000 dives vs. 3.70/10,000 dives), giving a RR of 1.57 (CI 0.71 to $3.47, \mathrm{p}=0.26$ ) (Table $3 \mathrm{~A}$ ).

As stated, of the 148 divers who returned the Final Questionnaire, only a minority were "non-blinded" (21 of 148, $18 \%)$. Analysis of only the "blinded" divers, unsurprisingly, does not change much to the previous analysis. For confirmed DCS, the incidence was $7.6 \%$ vs. $27.3 \%$, yielding an incidence of 1.8 and 5.46 DCS per 10,000 dives, respectively (for a Relative Risk of 3.02; CI 1.0502 to $8.7198, p=0.040$ ). For "all DCS, confirmed and possible," the incidence was $16.2 \%$ vs. $27.3 \%$, which amounts to 3.83 and 5.46 DCS per 10,000 dives (Relative Risk 1.42; CI 0.5616 to $3.6110, p=0.46)$ (Table 3B).

Regarding the types of symptoms of DCS, it is interesting to note that although cutaneous symptoms [cutis marmorata or livedo racemosa (Hartig et al., 2020)] and vestibular or cochlear DCS are most commonly associated with the presence of arterialized gas bubbles (PFO) (Germonpre et al., 1998; Wilmshurst et al., 2001; Cantais et al., 2003), it appears to be a symptom of (possible) DCS in RLS negative divers as well. Spinal cord decompression sickness also occurred (once) in shunt-positive divers.

Scrutiny of the DCS cases (for a more detailed description see the Supplementary Materials) revealed that all of the RLS positive divers that were treated with recompression had performed very deep dives, to a depth of $58,54,36$, and $65 \mathrm{~m}$, respectively (the $36 \mathrm{msw}$ dive was a closed-circuit rebreather CCR - dive with $50 \mathrm{~min}$ of bottom time).

The dives of those cases that were not treated with recompression were, likewise, more provocative than can be expected from recreational diving. One diver had severe vertigo and nausea after a $99 \mathrm{msw}$ dive on air. Other DCS cases in

TABLE 3A | Decompression sickness events (all divers).

\begin{tabular}{|c|c|c|c|}
\hline \multirow[b]{2}{*}{ n } & \multirow{2}{*}{$\frac{\text { RLS Positive }}{28}$} & \multirow{2}{*}{$\frac{\text { RLS Negative }}{120}$} & \multirow[t]{2}{*}{ p-value } \\
\hline & & & \\
\hline Confirmed DCS (n) & 8 & 10 & \\
\hline DCS (\%) & 28.6 & 8.3 & \\
\hline DCS incidence per 10,000 dives & 5.16 & 1.95 & 0.039 \\
\hline Possible DCS (n) & 1 & 9 & \\
\hline Total (confirmed + possible DCS) (\%) & 32.1 & 15.8 & \\
\hline Total incidence per 10,000 dives & 5.81 & 3.70 & 0.26 \\
\hline HBO treatment for DCS $(\mathrm{n})$ & 4 & 5 & \\
\hline Skin DCS (cutis marmorata) (n) & 4 & 9 & \\
\hline Vestibular/cochlear DCS (n) & 6 & 3 & \\
\hline Spinal cord DCS (n) & 1 & 5 & \\
\hline
\end{tabular}

TABLE 3B | Decompression sickness events ("blinded" divers only).

\begin{tabular}{lccccc}
\hline & RLS Positive & & RLS Negative & p-value \\
\cline { 2 - 2 } & $\mathbf{2 2}$ & & $\mathbf{1 0 5}$ & \\
\cline { 1 - 2 } Confirmed DCS (n) & 6 & & 8 & \\
DCS (\%) & 27.3 & & 7.6 & \\
DCS incidence per 10,000 dives & 5.46 & & 1.80 & 0.040 \\
Possible DCS (n) & 0 & & 9 & \\
Total (confirmed + possible DCS) (\%) & 27.3 & & 16.2 & \\
Total incidence per 10,000 dives & 5.46 & & 3.83 & 0.46 \\
HBO treatment for DCS (n) & 2 & & 3 & \\
Skin DCS (cutis marmorata) (n) & 3 & & 8 & \\
Vestibular/cochlear DCS (n) & 3 & & 2 & \\
Spinal cord DCS (n) & 1 & & 5 &
\end{tabular}


the RLS positive group were repetitive decompression dives, a square dive to $62 \mathrm{msw}$ for $68 \mathrm{~min}$ on CCR, square decompression cold water dives.

In the shunt-negative group, 4 treated DCS occurred after deep trimix technical dives or square decompression cold water dives ( 2 on CCR, 2 "open circuit"). Thirteen dives resulting in untreated post-dive symptoms were on average $40 \mathrm{msw}$ depth, which is at the limits of "recreational diving"; six of them were repetitive or decompression dives. Only two of those divers applied oxygen first aid, indicating that divers' denial is still very much present despite proper "education” (Lafere et al., 2017).

\section{DISCUSSION}

This is the first, and to our knowledge to date the only prospective evaluation of the Relative Risk (RR) of DCS when diving with RLS. It suggests that the risk for (confirmed) DCS is 2.65 times higher in divers with a RLS $(p=0.039)$. If we take into account all reported symptoms possibly associated with DCS, the RR is 1.57, which statistically is non-significant $(p=0.26)$. These figures are in line with previous, retrospective reports. However, it must be noted that most decompression sickness cases in the RLS positive group occurred after dives that are beyond reasonably defined recreational diving safety limits.

\section{Strengths and Weaknesses}

There are several strengths and weaknesses to our study.

First, the Carotid Doppler test detects right-to-left shunts without actual imaging of the inter-atrial septum. It is thus possible that some divers had in fact intrapulmonary shunts, not a PFO (Madden et al., 2015). However, in both validation studies (Blatteau, 1999; Germonpré et al., 1999) no pulmonary shunting was observed during transesophageal echocardiography, making the possibility of a significant "PFO-negative / Carotid Dopplerpositive" case number, very low. Furthermore, it is of little importance for divers whether RLS occurs through a PFO or a pulmonary shunt, as the end result (arterialized VGE) is the same. Finally, it would be anyhow improper to perform a PFO closure procedure without first verifying the morphology of the interatrial septum by echocardiography.

Secondly, at the end of 2019, when the data collection was definitively closed as it did not yield any further data, only 33.26 percent of all the divers had responded. According to our initial sample size calculations, the study would still be largely under-powered: with the assumed DCS incidence of 1 in 10,000, the power is only $40 \%$, However, the DCS incidence in our cohort was found to be much higher than the initially assumed incidence (2.53 per 10,000 dives). This reflects the incidence reported in other papers for "cold water sports diving" (Bove, 1998; Germonpre, 2006; Cialoni et al., 2017). If the sample size calculations are re-done using this actual incidence of DCS, then our study, as it is, has a $90 \%$ power. So even with the number of divers lower than expected, the study seems to have enough power to support our conclusions.

Thirdly, all confirmed and possible DCS cases were invited to perform a contrast transthoracic echocardiography. With proper saline contrast and straining maneuver technique, almost all PFO cases can be diagnosed on transthoracic echocardiography, obviating the need for transesophageal echocardiography (Wilmshurst, 2016). However, there are indications that patency of the Foramen Ovale may increase over time (Hagen et al., 1984; Germonpre et al., 2005). As the initial study period was already 7 years and by the end of the data collection, in some cases more than 15 years had passed since the initial Carotid Doppler test, a formal PFO detection would not necessarily be contributive: it would be expected that some divers who were initially "RLS negative" could now have become positive for PFO. In any case, only a minority declared to agree to this examination. This was thus not further pursued.

Fourth, $45 \%$ of our participants were, contrary to the protocol, not blinded to the results of the Carotid Doppler test. Analysis of these divers revealed that the response rate for non-blinded divers was significantly lower ( $14.5 \%$ vs. $48.57 \%$ in the blinded group), although the proportion of divers with DCS was similar in the non-blinded group than in the group that has been blinded ( $13.79 \%$ vs. $11.02 \%$ in the blinded group). At least two of the divers in the non-blinded group have decided to have their PFO closed subsequently to learning the Carotid Doppler test result. In one of the "non-blinded" groups, it was recorded that 6 out of 22 "RLS-positive" and 7 out of 49 "RLS-negative" divers had already suffered DCS prior to participating in the study. None of the divers of this group that responded to the final questionnaire (13 out of 71) reported DCS after having taken the Carotid Doppler test. This leads us to think that, indeed, if you inform a diver that a RLS has been detected, he/she might (subconsciously or consciously) adapt their diving behavior, be just a little bit more careful and may not have any or less decompression-induced vascular gas emboli. This has been published by Klingmann et al. (2012): by simply educating divers (with or without a PFO) about the risk for venous gas emboli and recommending "safe dive practices," the risk of subsequent decompression sickness can be reduced to almost zero, even in those divers with large PFOs. It was independently confirmed by Honěk et al. (2014b) Recommendations for conservative diving (also called "low-risk diving," "low-bubble diving") have been published by several diving safety organizations (Smart et al., 2015; Divers Alert Network, 2016; SUHMS, 2016), and basically consist of reducing the inert gas saturation during the dive by diving less deep, less long, or by breathing a gas with higher oxygen content ("Nitrox") while still using the dive computer as if air were breathed ("Nitrox on air profiles"). This increases the safety margin set by the dive computer (Souday et al., 2016) as these devices only take a limited number of parameters into account, and none of the physiological variations know to play a role in saturation and desaturation (Cialoni et al., 2017).

In this way, the mere fact of screening divers for PFO might induce a "safer" diving behavior, as an alternative to PFO closure. How long such behavior would persist, is not known. Also, those divers that have been informed that they have a RLS might be seeking PFO closure preventively (before any DCS has occurred) for instance because of their "risky" diving behavior, or even in anticipation of switching to more risky diving (technical diving) (Ljubkovic et al., 2010; Germonpre, 2015). 


\section{Strengths}

This study is the first, and to our knowledge, the only prospective study to date to observe a large number of divers who were blinded to their "RLS-status" over a large number of years, collecting data about their diving behavior and DCS incidents. Although not perfect, this study sought to minimize selection bias (divers were not financially or otherwise compensated for their participation), reporting bias (all instances of - possible - DCS were collected and reported) and diagnostic bias (the Carotid Doppler test detects both RLS through a PFO and through other shunting pathways, with minimal "observer variability").

It has been shown repeatedly that knowledge of a possible RLS influences the diving behavior (even after the shunt has been closed), therefore, a blinded study remains the only way to objectively ascertain the risk of DCS in diving attributable to a RLS (which is mostly through a PFO). Taking also into account the diving "habits" (deep, technical or purely recreational leisure diving) is likewise important, as the "base" DCS risk obviously will be different (Germonpre, 2006; Ljubkovic et al., 2010; Hubbard et al., 2018).

Secondly, this study provides valuable information to help divers take an informed decision whether or not to proceed to PFO closure. Indeed, more and more divers consult our and other diving medicine physicians' practice asking for a $\mathrm{PFO}$ detection test or seeking advice after a PFO has been fortuitously detected (i.e., without a history of DCS). That divers are ready to consider a (minimally, but still) invasive procedure in order to increase their (feeling of) security while diving, is an important observation. At least two of the divers in our study population have done so, both from the "non-blinded" group. Extrapolating this to all RLS positive divers, potentially up to $25 \%$ of those could be requesting PFO closure, even without ever having had DCS. Whether this is a valid preventive measure depends both on the risk involved in the closure procedure (short-, medium-, and long-term) and the degree of protection from DCS the procedure affords.

Several studies have described a reduction of arterial gas emboli in divers who have had their PFO closed as opposed to divers with PFO (Honěk et al., 2014a), even in a prospective manner. In view of the presumed pathophysiological mechanism, it seems logical that closing the PFO would reduce the risk of DCS after provocative (VGE-producing) dives.

A 5-year follow-up study after closure of PFO in Swiss divers has been published and is often cited as evidence that PFO closure is effective (Billinger et al., 2011). At first glance, the DCS incidence reduction in this report looks impressive $(0.5 / 10,000$ vs. 35.8/10,000 dives), but the absolute numbers of DCS cases are very low (4 in the "PFO" group, 1 in the "Closure" group over a 5-year period). In fact, if only one diver in the "PFO" group would have decided that his/her symptoms were not DCS, or if one diver in the "Closure" group would have declared that some vague symptom he/she had experienced were probably DCS, there would have been no statistical difference between those two groups. Moreover, divers who did not have their PFO closed (the "PFO" group) performed almost 50\% of their dives deeper than $40 \mathrm{msw}$, whereas only $30 \%$ of dives in the "Closure" group was deeper than $40 \mathrm{msw}$.
Divers with a PFO (closed or not) apparently did change their diving procedures somewhat after the diagnosis, by performing slightly less of the deep ( $>40 \mathrm{msw}$ ) dives and choosing more often Nitrox as a breathing gas (although it was not specified whether they used "Nitrox" or "air" decompression procedures).

In other studies it was also reported that even though the PFO was closed, a significant number of divers adopted a more conservative diving attitude (Koopsen et al., 2018; Anderson et al., 2019; Vanden Eede et al., 2019). A recent report (Anderson et al., 2019) described a better reduction of DCS incidence after PFO closure compared to "conservative diving," but major methodological flaws have been pointed out by an accompanying editorial (Wilmshurst, 2019).

A recent study (Vanden Eede et al., 2019) describing the follow-up of 59 PFO closures over a 10-year period in a Belgian diving population has reported a significant number of recurring DCS (15\%) despite the PFO closure. These occurred either because the PFO had not been completely closed, or because of extremely provocative dives. Also, the procedure had an incidence of complications and side effects comparable to other reports (Pristipino et al., 2019).

Honěk et al. (2021) very recently reported the results of a risk stratification strategy study whereby divers, screened for PFO were offered the choice between PFO closure or "conservative diving" in case of high-grade PFO. Although both groups had a decreased incidence of DCS during the follow-up period of 6.5 years (the "closure group" approaching the DCS incidence of those divers without PFO), the "highgrade PFO - conservative diving" group still had a higher incidence. The depths and dive profiles attained by all of these divers, however, are to be considered quite provocative for recreational diving (depths to more than 40 meters, on air or nitrox); moreover, the "conservative diving" recommendations apparently consisted only of recommending dive computer"no-decompression diving" to a maximum of $40 \mathrm{~m}$. It appears from the Supplementary Data of this paper that only $75.5 \%$ of the "high-grade PFO" divers actually followed these recommendations.

Closing a PFO can thus be considered effective in reducing the risk of DCS but should not be considered a "free ticket" to unrestricted provocative diving. Also, long-term complications of the closure procedure are reported sporadically, and their incidence in follow-up studies is significant (Abaci et al., 2013; Merkler et al., 2017). On the other hand, the effectiveness of recommending "conservative diving practice" is dependent on a continued diver education and could benefit from a "cultural change" in the diver community. For divers who cannot (e.g., professional divers) or do not wish to adopt (e.g., deep, technical divers) conservative diving practice, closing a PFO might, however, reduce the risk for DCS by a factor three.

\section{CONCLUSION}

Based on this prospective analysis of divers blinded to their "RLS-status," we suggest a Relative Risk of DCS for recreational 
diving with a RLS to be 1.42 (all DCS) to 3.02 (confirmed DCS). However, the absolute risk for confirmed DCS is low $(2.53 / 10,000$ dives all divers, 1.80/10,000 RLS negative and 5.46/10,000 RLS positive) and even more, most of these DCS cases happen outside what we would consider "safe recreational diving."

We therefore confirm the need for comprehensive evaluation and expert counseling of each diver presenting with DCS and PFO and seeking advice whether this PFO should be closed (Germonpre, 2015; Smart et al., 2015; Wilmshurst, 2019). Any recommendation on "conservative diving" should be given according to internationally published "low-bubble" guidelines, not simply relying on the dive computer to make "nodecompression dives." Systematic screening of recreational divers for RLS or PFO, in the absence of a history of DCS, remains in our opinion not warranted.

\section{DATA AVAILABILITY STATEMENT}

The original contributions presented in the study are included in the article/Supplementary Material, further inquiries can be directed to the corresponding author.

\section{ETHICS STATEMENT}

The studies involving human participants were reviewed and approved by ethical committee approval was obtained (Bioethics Committee of the Belgian Defense Force Medical Staff, 2003), and divers signed an informed consent form prior to the

\section{REFERENCES}

Abaci, A., Unlu, S., Alsancak, Y., Kaya, U., and Sezenoz, B. (2013). Short and long term complications of device closure of atrial septal defect and patent foramen ovale: meta-analysis of 28,142 patients from 203 studies. Catheter. Cardiovasc. Interv. 82, 1123-1138. doi: 10.1002/ccd.24875

Anderson, G., Ebersole, D., Covington, D., and Denoble, P. J. (2019). The effectiveness of risk mitigation interventions in divers with persistent (patent) foramen ovale. Diving Hyperb. Med. 49, 80-87. doi: 10.28920/dhm49.2. 80-87

Attaran, R. R., Ata, I., Kudithipudi, V., Foster, L., and Sorrell, V. L. (2006). Protocol for optimal detection and exclusion of a patent foramen ovale using transthoracic echocardiography with agitated saline microbubbles. Echocardiography 23, 616-622. doi: 10.1111/j.1540-8175.2006.00272.x

Billinger, M., Zbinden, R., Mordasini, R., Windecker, S., Schwerzmann, M., Meier, B., et al. (2011). Patent foramen ovale closure in recreational divers: effect on decompression illness and ischaemic brain lesions during long-term follow-up. Heart 97, 1032-1937. doi: 10.1136/heartjnl-2011-300436

Blatteau, J.-E. (1999). Carotid artery Doppler as a non-invasive method for rightto-left shunt screening. Study about 200 patients versus trans-oesophageal contrast echocardiography. Bull. Med. Sub. Hyp. 9, 97-103.

Bove, A. A. (1998). Risk of Decompression sickness with patent foramen ovale. Undersea Hyperb. Med. 25, 175-178.

Cantais, E., Louge, P., Suppini, A., Foster, P. P., and Palmier, B. (2003). Right-toleft shunt and risk of decompression illness with cochleovestibular and cerebral symptoms in divers: case control study in 101 consecutive dive accidents. Crit. Care Med. 31, 84-88. doi: 10.1097/00003246-200301000-00013

Cialoni, D., Pieri, M., Balestra, C., and Marroni, A. (2017). Dive risk factors, gas bubble formation, and decompression illness in recreational SCUBA diving: analysis of DAN Europe DSL data base. Front. Psychol. 8:1587. doi: 10.3389/ fpsyg.2017.01587 testing. Each testing was performed individually, subjects going through the informed consent process one at a time. The patients/participants provided their written informed consent to participate in this study.

\section{AUTHOR CONTRIBUTIONS}

All authors listed have made a substantial, direct and intellectual contribution to the work, and approved it for publication.

\section{ACKNOWLEDGMENTS}

We would like to express our gratitude to all the divers and the investigators having participated in the data collection (Thomas Soliman, Françoise Jadoul, Peter Mueller, Ronald Luecke, Gerling Jaeger, Luca Torcello, Jürg Wendling, Tudor Codreanu, Frans Cronje, and Hermie Britz). Also, the recruitment efforts though the Diver Alert Network website and publications were highly appreciated. There was no external funding for this study and no financial benefit for any of the divers or investigators.

\section{SUPPLEMENTARY MATERIAL}

The Supplementary Material for this article can be found online at: https://www.frontiersin.org/articles/10.3389/fphys. 2021.763408/full\#supplementary-material

Divers Alert Network (2016). Guidelines for Patent Foramen Ovale and Diving. Durham, NC: Divers Alert Network.

Germonpre, P. (2006). The medical risks of underwater diving and their control. Int. Sportmed J. 7, 1-15.

Germonpre, P. (2015). Persistent (patent) foramen ovale (PFO): implications for safe diving. Diving Hyperb. Med. 45, 73-74.

Germonpre, P., and Balestra, C. (2004). Risk of decompression illness among 230 divers in relation to the presence and size of patent foramen ovale. Eur. Heart J. 25, 2173-2174. doi: 10.1016/j.ehj.2004.07.042

Germonpre, P., Dendale, P., Unger, P., and Balestra, C. (1998). Patent foramen ovale and decompression sickness in sports divers. J. Appl. Physiol. 84, 16221626. doi: 10.1152/jappl.1998.84.5.1622

Germonpre, P., Hastir, P., Dendale, P., Marroni, A., Nguyen, A.-F., and Balestra, C. (2005). Evidence for increasing patency of the foramen ovale in divers. Am. J. Cardiol. 95, 912-915. doi: 10.1016/j.amjcard.2004.12.026

Germonpré, P., Balestra, C., Dendale, P., Unger, P., and Vandenhoven, G. (1999). "Carotid artery doppler as a minimally invasive screening method for patency of the foramen ovale," in Proceedings of the EUBS Meeting 1999 (26-29 August), Eilat.

Gin, K. G., Huckell, V. F., and Pollick, C. (1993). Femoral vein delivery of contrast medium enhances transthoracic echocardiographic detection of patent foramen ovale. J. Am. Coll. Cardiol. 22, 1994-2000. doi: 10.1016/0735-1097(93)90789-4

Hagen, P. T., Scholz, D. G., and Edwards, W. D. (1984). Incidence and size of patent foramen ovale during the first 10 decades of life: an autopsy study of 965 normal hearts. Mayo Clin. Proc. 59, 17-20. doi: 10.1016/S0025-6196(12)60336-X

Hartig, F., Reider, N., Sojer, M., Hammer, A., Ploner, T., Muth, C. M., et al. (2020). Livedo racemosa-the pathophysiology of decompression-associated cutis marmorata and right/left shunt. Front. Physiol. 11:994. doi: 10.3389/fphys. 2020.00994

Honěk, J., Šrámek, M., Honěk, T., Tomek, A., Šefc, L., Januška, J., et al. (2020). Patent foramen ovale closure is effective in divers: long-term results from the 
DIVE-PFO registry. J. Am. Coll. Cardiol. 76, 1149-1150. doi: 10.1016/j.jacc. 2020.06.072

Honěk, J., Šrámek, M., Honěk, T., Tomek, A., Šefc, L., Januška, J., et al. (2021). Screening and risk stratification strategy reduced decompression sickness occurrence in divers with patent foramen ovale. J. Am. Coll. Cardiol. S1936878X, 00509-X. doi: 10.1016/j.jcmg.2021.06.019

Honěk, J., Šrámek, M., Šefc, L., Januška, J., Fiedler, J., Horváth, M., et al. (2014a). Effect of catheter-based patent foramen ovale closure on the occurrence of arterial bubbles in scuba divers. J. Am. Coll. Cardiol. Interv. 7, 403-408. doi: 10.1016/j.jcin.2013.12.199

Honěk, J., Šrámek, M., Šefc, L., Januška, J., Fiedler, J., Horváth, M., et al. (2014b). Effect of conservative dive profiles on the occurrence of venous and arterial bubbles in divers with a patent foramen ovale: a pilot study. Int. J. Cardiol. 176, 1001-1002. doi: 10.1016/j.ijcard.2014.04.218

Hubbard, M., Davis, F. M., Malcolm, K., and Mitchell, S. J. (2018). Decompression illness and other injuries in a recreational dive charter operation. Diving Hyperb. Med. 48, 218-223. doi: 10.28920/dhm48.4.218-223

Johansson, M., Eriksson, P., Wallentin Guron, C., and Dellborg, M. (2010). Pitfalls in diagnosing PFO: characteristics of false- negative contrast injections during transesophageal echocardiography in patients with patent foramen ovale. J. Am. Soc. Echocardiogr. 23, 1136-1142. doi: 10.1016/j.echo.2010.08.004

Klingmann, C., Rathmann, N., Hausmann, D., Bruckner, T., and Kern, R. (2012). Lower risk of decompression sickness after recommendation of conservative decompression practices in divers with and without vascular right-to-left shunt. Diving Hyperb. Med. 42, 146-150.

Koopsen, R., Stella, P. R., Thijs, K. M., and Rienks, R. (2018). Persistent foramen ovale closure in divers with a history of decompression sickness. Neth. Heart J. 26, 535-539. doi: 10.1007/s12471-018-1153-x

Lafere, P., Balestra, C., Caers, D., and Germonpre, P. (2017). Patent foramen ovale (PFO), personality traits, and iterative decompression sickness. retrospective analysis of 209 cases. Front. Psychol. 8:1328. doi: 10.3389/fpsyg.2017.01328

Ljubkovic, M., Marinovic, J., Obad, A., Breskovic, T., Gaustad, S. E., and Dujic, Z. (2010). High incidence of venous and arterial gas emboli at rest after trimix diving without protocol violations. J. Appl. Physiol. 109, 1670-1674. doi: 10. 1152/japplphysiol.01369.2009

Madden, D., Ljubkovic, M., and Dujic, Z. (2015). Intrapulmonary shunt and SCUBA diving: another risk factor? Echocardiography 32(Suppl. 3), S205-S210. doi: $10.1111 /$ echo. 12815

Merkler, A. E., Gialdini, G., Yaghi, S., Okin, P. M., Iadecola, C., Navi, B. B., et al. (2017). Safety outcomes after percutaneous transcatheter closure of patent foramen ovale. Stroke 48, 3073-3077. doi: 10.1161/STROKEAHA.117.018501

Moon, R. E., Camporesi, E. M., and Kisslo, J. A. (1989). Patent foramen ovale and decompression sickness in divers. Lancet 1, 513-514. doi: 10.1016/S01406736(89)90064-0

Pristipino, C., Germonpre, P., Toni, D., Sievert, H., Meier, B., D’Ascenzo, F., et al. (2021). European position paper on the management of patients with patent foramen ovale. Part II-decompression sickness, migraine, arterial deoxygenation syndromes and select high-risk clinical conditions. Eur. Heart J. 42, 1545-1553. doi: 10.1093/eurheartj/ehaa 1070

Pristipino, C., Sievert, H., D’Ascenzo, F., Mas, J. L., Meier, B., Scacciatella, P., et al. (2019). European position paper on the management of patients with patent foramen ovale. General approach and left circulation thromboembolism. EuroIntervention 14, 1389-1402. doi: 10.4244/EIJ-D-18-00622

Smart, D., Mitchell, S., Wilmshurst, P., Turner, M., and Banham, N. (2015). Joint position statement on persistent foramen ovale (PFO) and diving. South Paci fi c Underwater Medicine Society (SPUMS) and the United Kingdom Sports Diving Medical Committee (UKSDMC). Diving Hyperb. Med. 45, 129-131.
Souday, V., Koning, N. J., Perez, B., Grelon, F., Mercat, A., Boer, C., et al. (2016). Enriched air nitrox breathing reduces venous gas bubbles after simulated SCUBA diving: a double-blind cross-over randomized trial. PLoS One 11:e0154761. doi: 10.1371/journal.pone.0154761

Steffensmeier, D., Albrecht, R., Wendling, J., Melliger, R., Spahn, D. R., Stein, P., et al. (2017). Specialist advice may improve patient selection for decompression therapy following diving accidents: a retrospective observational study. Scand. J. Trauma Resusc. Emerg. Med. 25:101. doi: 10.1186/s13049-017-0447-0

SUHMS (2016). SUHMS Guidelines for Diving with a Patent Foramen Ovale. Available online at: https://suhms.org/pfo_bild/ (accessed November 11, 2016)

Sykes, O., and Clark, J. E. (2013). Patent foramen ovale and scuba diving: a practical guide for physicians on when to refer for screening. Extrem. Physiol. Med. 2:10. doi: 10.1186/2046-7648-2-10

Torti, S. R., Billinger, M., Schwerzmann, M., Vogel, R., Zbinden, R., Windecker, S., et al. (2004). Risk of decompression illness among 230 divers in relation to the presence and size of patent foramen ovale. Eur. Heart J. 25, 1014-1020. doi: 10.1016/j.ehj.2004.04.028

Vanden Eede, M., Van Berendoncks, A., De Wolfe, D., De Maeyer, C., Vanden Eede, H., and Germonpre, P. (2019). Percutaneous closure of patent foramen ovale for the secondary prevention of decompression illness in sports divers: mind the gap. Undersea Hyperb. Med. 46, 625-632. doi: 10.22462/10.12.2019.6

Vann, R. D., Moon, R. E., Freiberger, J. J., Denoble, P. J., Dear, G. L., Stolp, B. W., et al. (2008). Decompression illness diagnosis and decompression study design. Aviat. Space Environ. Med. 79, 797-798. doi: 10.3357/ASEM.2316.2008

Wendling, J., Balestra, C., and Germonpre, P. (2001). Is screening for foramen ovale feasibe? SPUMS J. 31, 85-89.

Wilmshurst, P. (2016). Detection of a persistent foramen ovale using echocardiography. Diving Hyperb. Med. 46, 47-49.

Wilmshurst, P. (2019). Risk mitigation in divers with persistent (patent) foramen ovale. Diving Hyperb. Med. 49, 77-78. doi: 10.28920/dhm49.2.77-78

Wilmshurst, P., Pearson, M., Walsh, K., Morrison, W., and Bryson, P. (2001). Relationship between right-to-left shunts and cutaneous decompression illness. Clin. Sci. (Lond.) 100, 539-542. doi: 10.1042/CS20000296

Wilmshurst, P. T., Byrne, J. C., and Webb-Peploe, M. M. (1989). Relation between interatrial shunts and decompression sickness in divers. Lancet 2, 1302-1306. doi: 10.1016/S0140-6736(89)91911-9

Wilmshurst, P. T., Pearson, M. J., Walsh, K. P., and Morrison, W. L. (2003). In clinical practice transoesophageal echocardiography usually fails to detect large persistent foramen ovale (Abstract). Heart 89, 38-39.

Conflict of Interest: The authors declare that the research was conducted in the absence of any commercial or financial relationships that could be construed as a potential conflict of interest.

Publisher's Note: All claims expressed in this article are solely those of the authors and do not necessarily represent those of their affiliated organizations, or those of the publisher, the editors and the reviewers. Any product that may be evaluated in this article, or claim that may be made by its manufacturer, is not guaranteed or endorsed by the publisher.

Copyright (c) 2021 Germonpré, Lafère, Portier, Germonpré, Marroni and Balestra. This is an open-access article distributed under the terms of the Creative Commons Attribution License (CC BY). The use, distribution or reproduction in other forums is permitted, provided the original author(s) and the copyright owner(s) are credited and that the original publication in this journal is cited, in accordance with accepted academic practice. No use, distribution or reproduction is permitted which does not comply with these terms. 